\title{
Original
}

\section{Declaración de embarazo del personal sanitario del hospital universitario Ramón y Cajal. Año 2009}

\author{
Pregnancy declaration of healthcare workers \\ of the Ramon y Cajal university hospital. Year 2009
}

\section{Gian C. Navarro Chumbes; Ignacio Sánchez-Arcilla Conejo; Marina Fernández Escribano}

Servicio de Prevención de Riesgos Laborales del Hospital Universitario Ramón y Cajal. Madrid. España.

\section{Recibido: 25-05-11}

Aceptado: 03-06-11

\author{
Correspondencia \\ D. Gian C. Navarro Chumbes \\ Servicio de Prevención de Riesgos Laborales \\ Hospital Universitario Ramón y Cajal \\ Carretera de Colmenar Viejo, Km 9, 100 \\ 28049 Madrid. España. \\ Tfno: 669550374 \\ E-mail: gcnavarro@yahoo.com / gcnavarro@hotmail.com
}

Resumen

Introducción: según la Ley 31/1995 del 8 de noviembre de Prevención de Riesgos Laborales, Capítulo III artículo 26 "Protección de la Maternidad", el empresario deberá adoptar las medidas para evitar la exposición a riesgos en mujeres embarazadas y en periodos de lactancia.

Objetivo: conocer las características del personal sanitario que declaró su embarazo al Servicio de Prevención de Riesgos Laborales (SPRL).

Material y Métodos: el diseño del estudio es transversal, los datos fueron obtenidos del personal sanitario gestante que declaró su embarazo al SPRL durante el año 2009; se realizó análisis de estadística descriptiva de media +/- desviación estándar, así como porcentajes. Se consideraron significativas aquellas con un valor de significación $<0.05$.

Resultados: declararon su embarazo 50 personas; el promedio de edad fue $33.10+/-3.18$ años. El grupo etáreo que más declaró fue el de 30-34 años (52\%); el promedio de semanas de embarazo al declarar fue de 12.01 +/- 5.18; la categoría profesional que más declaró fue el de DUE (Diplomado universitario en enfermería) $24.48 \%$.

Conclusiones: de acuerdo con los resultados obtenidos y contrastados con los de la Comunidad de Madrid deberían plantearse otros estudios para averiguar si hay infradeclaración del embarazo y sus motivos.

Med Segur Trab (Internet) 2011; 57 (223) 147-153

Palabras clave: trabajador de salud, declaración, embarazo.

Abstract

Introduction: according to the Labour Risks Prevention Law 31/1995 of November the 8th, Chapter III, article 26 "Protection of Maternity", the employer must carry out all precautions in order to avoid exposure to risks in pregnant women as well as breastfeeding women. 
Objective: know all characters of healthcare workers that declared their pregnancy to the Labour Risks Prevention Service (LRPS).

Method and materials: the study design is transversal; all data was obtained from the healthcare workers that declared their pregnancy to the LRPS in 2009; a descriptive statistic analysis of media +/standard deviation and percentages was done. A value of $\mathrm{p}<0.05$ was considered statistically significant.

Results: 50 persons declared their pregnancy; age average was 33.10+/-3.18 years. The age group that most frequent declared was 30-34 years (52\%), the average week of pregnancy at the moment of declaring was $12.01+/-5.18$; the professional category that most frequently declared was UNQ (University Nursing Qualified) $24.48 \%$.

Conclusions: according to the results obtained and after contrasting them with the data of the Community of Madrid, other studies must be designed to find out if there is underreporting of pregnancy and its causes.

Med Segur Trab (Internet) 2011; 57 (223) 147-153

Key words: bealthcare worker, declaration, pregnancy. 


\section{INTRODUCCIÓN}

La proporción de mujeres que trabajan y que lo continúan haciendo durante el embarazo va en aumento. El embarazo no es una enfermedad, sino un aspecto más en la vida de una mujer que en muchos casos debe compaginar con su actividad laboral, y que en ocasiones hay un riesgo para el embarazo ${ }^{1}$.

La legislación vigente sobre la protección del embarazo y de la maternidad se centra básicamente en los agentes físicos y químicos como la exposición a benceno, a plomo, radiaciones ionizantes, etc., siendo en la actualidad muy escasa la referente a carga de trabajo.

La consideración de sensible a la mujer trabajadora embarazada se considera en España en la Ley 39/1999 del 5 de noviembre para la conciliación entre la vida familiar y laboral de las personas trabajadoras en la que además se amplían las prestaciones económicas de la Ley General de la Seguridad Social en la que incluye la prestación económica por riesgo durante el embarazo; posteriormente la Ley Orgánica 3/2007 del 22 de marzo establece la igualdad efectiva de mujeres y hombres ${ }^{2}$. Sin embargo debido a la experiencia adquirida en los últimos años con la finalidad de facilitar la identificación de agentes, procedimientos y condiciones de trabajo, así como garantizar una aplicación más efectiva de las disposiciones de protección de la maternidad se modifica el Real Decreto 39/1997 de 17 de enero mediante el Real Decreto 298/2009, de 6 de marzo, en relación con la aplicación de medidas para promover la mejora de la seguridad y de la salud en el trabajo de la trabajadora embarazada, que haya dado a luz o en periodo de lactancia ${ }^{3}$.

En ocasiones el embarazo es patológico por causa clínica interfiriendo en el desarrollo laboral provocando la ausencia de la mujer al trabajo (incapacidad temporal).

La legislación española en lo referente a Seguridad Social ha establecido dos subsidios para cada una de las situaciones en la que es necesaria la ausencia de la mujer de su puesto de trabajo. Si la situación se atribuye a la situación clínica del embarazo (embarazo de riesgo) puede ser susceptible a incapacidad temporal; pero si la situación se atribuye a la existencia de un agente nocivo en el trabajo (riesgo) se debe valorar como riesgo durante el embarazo. Si ambas situaciones se dan a la vez debe valorarse la predominancia del factor clínico o laboral y por consiguiente establecer el subsidio correspondiente ${ }^{4}$.

\section{JUSTIFICACIÓN Y OBJETIVOS}

La Ley 31/1995 del 8 de noviembre de Prevención de Riesgos Laborales en el Capítulo III del artículo 26 respecto a "Protección de la maternidad" establece que como resultado de la evaluación de riesgos el empresario deberá adoptar las medidas necesarias para evitar la exposición a riesgos en mujeres embarazadas y en periodos de lactancia, incluyendo, si es necesario, la no realización de trabajo nocturno y/o a turnos. Cuando estas medidas no sean aplicables, se facilitará una nueva tarea o puesto de trabajo compatible con el estado de la mujer embarazada, hasta que ésta pueda volver a su trabajo habitual. Si no hubiere ningún puesto compatible, la trabajadora puede ser destinada a otro grupo o categoría, conservando el derecho al conjunto de retribuciones de su puesto de origen. Todas estas disposiciones son asimismo aplicables en el periodo de lactancia. El capítulo VII artículo 48 de la Ley 31/1995 contempla como infracción muy grave el no observar las normas específicas en materia de protección de la seguridad y salud de las trabajadoras durante los periodos de embarazo y lactancia ${ }^{1}$.

Con la finalidad de iniciar el procedimiento tal y como establece la Ley 31/1995 es necesaria la comunicación del embarazo de la trabajadora a la empresa al SPRL. 
En el SPRL del Hospital Universitario Ramón y Cajal se están registrando las comunicaciones del embarazo del personal sanitario desde el año 2003, lo cual aporta información importante en el ámbito de la Medicina del Trabajo. Las declaraciones del embarazo han ido aumentando en el transcurso de los años al haberse ido implementando la cultura de la declaración y protección del embarazo.

El objetivo principal de nuestro estudio es conocer las características del personal sanitario que ha declarado su embarazo al SPRL, teniendo en cuenta factores como edad, trimestre de gestación al momento de declarar el embarazo, distribución por categoría profesional, distribución por servicio, distribución por riesgos y por el número de visitas hechas al SPRL después de declarar la gestación.

\section{MATERIAL Y MÉTODOS}

El diseño del estudio es transversal; los datos del personal sanitario gestante que durante el año 2009 declaró su embarazo se obtuvieron de los registros de embarazo existente en el SPRL del Hospital Universitario Ramón y Cajal.

A partir de estos datos se elaboró una base de datos en Excel, posteriormente se codificó la información obtenida y para el análisis se utilizó el paquete estadístico SPSS (Statistical Package for Social Sciences) versión 15.0 para Windows. Se realizó un análisis de estadística descriptiva para las variables cuantitativas de media \pm desviación estándar, así como porcentajes. Se consideraron diferencias significativas aquellas con un valor de significación inferior a $0.05(\mathrm{p}<0.05)$.

\section{RESULTADOS}

Durante el año 2009 acudieron a declarar el embarazo 50 personas, el promedio de edad fue de 33.10 +/- 3.18 años pudiendo observarse en la Figura 1 la distribución por grupos etáreos.

Figura 1. Distribución por edad.

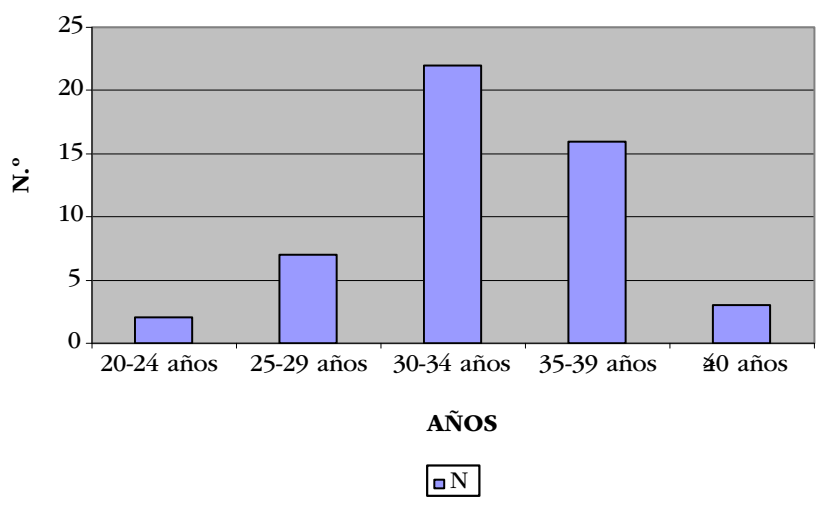

El promedio de semanas de embarazo al momento de declarar fue de $12.01+/-5.18$, encontrándose que el $61.22 \%$ lo hizo en el primer trimestre de embarazo, el $36.73 \%$ en el segundo trimestre de embarazo y el $2.04 \%$ en el tercer trimestre de embarazo.

Respecto a la categoría profesional y servicio que desempeña cada gestante al momento de declarar se observa en le Figura 2 y Figura 3 que en su mayoría eran DUEs y pertenecían a Servicios Médicos. 
Figura 2. Distribución por categoría profesional.

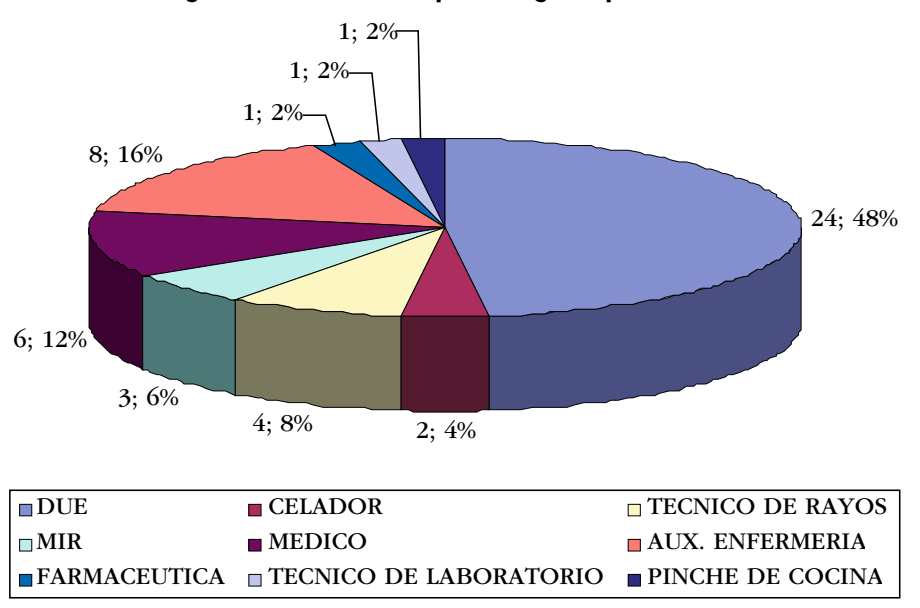

Figura 3. Distribución por servicio.

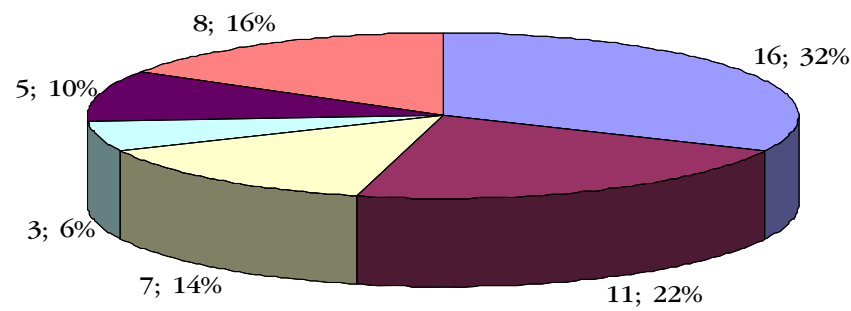

\begin{tabular}{|lll|}
\hline S.MÉDICOS & $\square$ S.QUIRÚRGICOS & $\square$ HOSPITALIZACIÓN \\
$\square$ URGENCIAS & $\square$ UVI & $\square$ SERVICIOS CENTRALES \\
\hline
\end{tabular}

El número de riesgos a los que estaba expuesta cada gestante en promedio era $2.3+/-0.91$ siendo mayormente a riesgos biológicos (72 \%) y a nocturnicidad/yurnicidad (34\%). Ver Figura 4.

Respecto al número de visitas realizadas al SPRL tras declarar el embarazo el promedio era de $1.12+/-0.21$.

Figura 4. Distribución por riesgos.

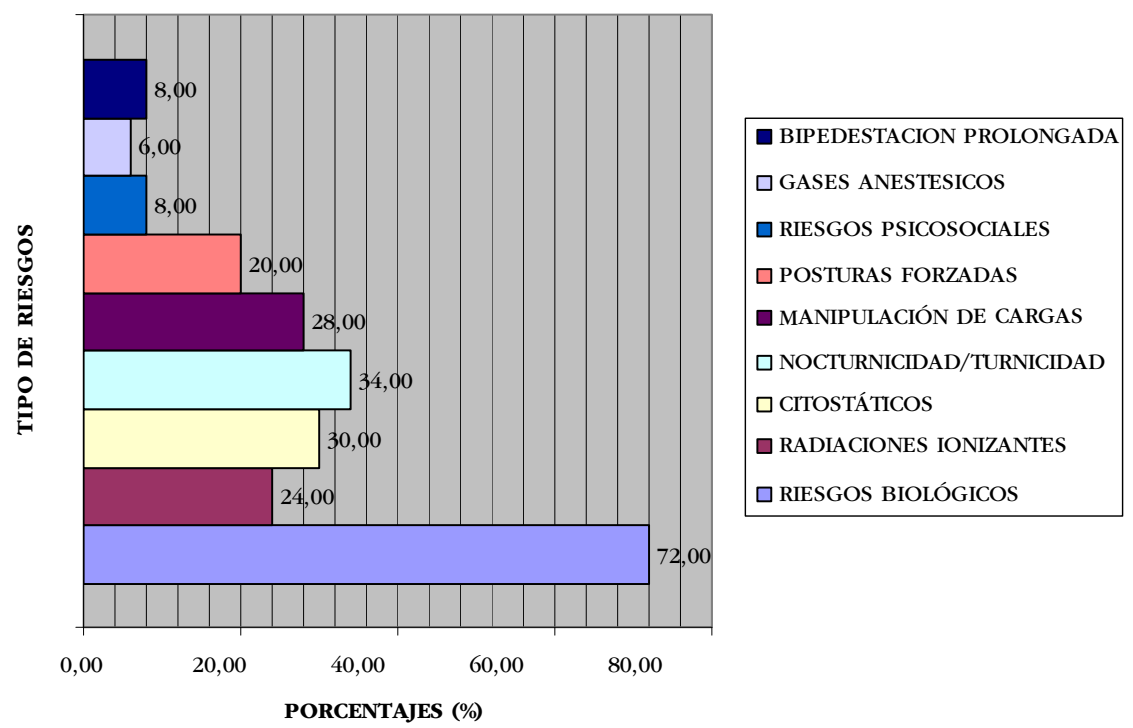




\section{DISCUSIÓN Y CONCLUSIONES}

Para que el empresario pueda cumplir con la Ley de "Protección de Maternidad" es imprescindible conocer la existencia del embarazo de su personal, por ello es importante que la trabajadora embarazada o lactante lo comunique a fin de adoptar las medidas necesarias para evitar su exposición a riesgos laborales, incluyendo, si es necesario, la no realización de trabajo nocturno y/o a turnos.

Según los datos obtenidos del programa MEDTRA-SETRA, actualización del día 6 de octubre del 2010, tenemos que en el Hospital Ramón y Cajal hay 6372 trabajadores, 4750 (74.54\%) son trabajadoras, y en edad reproductiva hay 2363 (49.75\%).

El promedio de edad de las trabajadoras que declararon su embarazo es de $33.10+/-3.18$ años, resultado muy similar al promedio de edad de todas las trabajadoras en edad reproductiva que laboran en el hospital 31.85 +/- 5.60 años; siendo el rango de edad de las trabajadoras que declararon su embarazo en el SPRL durante el año 2009 fue de 23 a 41 años que declaró. Asimismo se aprecia que la edad media de las trabajadoras que declararon su embarazo es mayor que la edad media de maternidad en Madrid (31.29 años) según los datos del Instituto Nacional de Estadística (INE).

Durante el año 2009 en el SPRL se registraron 50 declaraciones, y observó que el grupo etáreo que más declaró el embarazo fue el de 30-34 años, (22 trabajadoras, 44 \% de las que declararon su embarazo), seguido del grupo 35-39 años (16 trabajadoras, $32 \%$ de las que declararon su embarazo) y luego del grupo 25-29 años (7 trabajadoras, 14 \% de las que declararon su embarazo).

Al contabilizar el número de los grupos etáreos que declararon el embarazo respecto a los mismos grupos etáreos del total de las trabajadoras de todo el hospital en edad reproductiva tenemos que los porcentajes correspondientes fueron los siguientes: grupo 30-34 años $4.45 \%$ (22/494), grupo 35-39 años $3.35 \%$ (16/478) y el grupo 25-29 años $1.31 \%$ (7/536); y al ser comparado con las tasas de fecundidad de los mismos grupos etáreos de la Comunidad de Madrid según los datos del INE tenemos lo siguiente: 30-34 años $10.72 \%, 35-39$ años $7.19 \%$ y 25-29 años $6.28 \%$; podemos apreciar que los porcentajes de declaración de embarazo del Hospital Universitario Ramón y Cajal respecto a los de la Comunidad de Madrid se mantienen en el mismo orden, sin embargo siendo menor la declaración en el Hospital, lo que podría interpretarse como menor número de declaraciones del embarazo o al menor número de embarazos. En cualquier caso se deberían estudiar las causas de ambos supuestos, pudiendo especularse que las razones podrían ser porque lo desconocen, porque no hay interés ni ganancia secundaria por parte de la interesada.

Hemos encontrado que el momento de declarar el embarazo es mayormente en el primer trimestre (61.22\%) lo que es esperable porque el mayor riesgo existente que se produce para el embarazo se da en las primeras semanas; sin embargo encontramos que el $36.73 \%$ lo declara en el segundo trimestre y $2.04 \%$ en el tercer trimestre por lo que es importante conocer el porqué de la demora en la declaración y si es que se han tomado las medidas preventivas oportunas ya sea a través de su médico de cabecera, su supervisor(a) o por ella misma con la finalidad de evitar el riesgo.

La categoría profesional que mayormente declaró el embarazo fue la correspondiente a las DUE $24.48 \%$ seguido por las auxiliares de Enfermería $16 \%$ y luego por los médicos $12 \%$; estos resultados se relacionan con que es la categoría profesional más numerosa ya que en el hospital el $30.82 \%$ (1464) de las trabajadoras es DUE, seguido de las auxiliares de Enfermería 24.11 \% (1145) y los médicos 8.86 \% (421); sin embargo deberíamos averiguar si la mayor declaración es porque corresponde al grupo más numeroso en el Hospital o es que hay otras causas que deberíamos identificar mediante otros estudios.

Observamos que al analizar los servicios a los que pertenecen las trabajadoras que han declarado el embarazo tenemos que los 3 más predominantes son: servicios médicos 16 (32\%), servicio quirúrgicos 11 (22\%) y hospitalización 7 (14\%) respectivamente; 
asimismo tenemos que se mantiene el mismo orden al comparar el mismo número de trabajadoras que han declarado el embarazo de los servicios mencionados respecto al total de trabajadoras correspondientes a los mismos servicios mencionados tenemos: servicios médicos $3.65 \%$ (16/438), servicios quirúrgicos 2.18 \% (11/504) y hospitalización $1.38 \%(7 / 559)$.

Los tres riesgos a los que más estuvieron expuestas las trabajadoras que declararon su embarazo fueron riesgos biológicos (72 \%), nocturnicidad/turnicidad (34 \%) y citostáticos (30\%), lo cual era esperado debido a que la categoría profesional que más acudió a declarar al SPRL correspondía a las DUE.

Asimismo con la finalidad de evaluar el(los) riesgo(s) de las trabajadoras que declaran el embarazo y cuando las condiciones lo requieran aplicar restricción de tareas, adaptación del puesto o el cambio de puesto si es necesario, las trabajadoras deben acudir nuevamente al SPRL por cada trimestre de embarazo transcurrido debido a que los riesgos se van modificando a medida que transcurre el embarazo; ${ }^{5}$ asimismo las trabajadoras deben comunicar cómo evoluciona el embarazo, si han surgido alteraciones, etc; sin embargo encontramos que sólo el $12 \%$ acudió al SPRL una vez más luego de declarar el embarazo.

Ante lo encontrado es importante investigar las causas de la no declaración del embarazo al SPRL y por qué un gran porcentaje espera hasta el segundo trimestre de embarazo para acudir al SPRL a declararlo, de esa manera corregir las causas y por consiguiente tomar las medidas necesarias para evitar la exposición a riesgos en embarazadas y posteriormente durante la lactancia.

\section{REFERENCIAS BIBLIOGRÁFICAS}

1. NTP 413: Carga de trabajo y embarazo. Instituto Nacional de Seguridad e Higiene del Trabajo. Ministerio de Trabajo y Asuntos Sociales. España.

2. NTP 612: Protección y promoción de la salud reproductiva: funciones del personal sanitario del servicio de prevención. Instituto Nacional de Seguridad e Higiene del Trabajo. Ministerio de Trabajo y Asuntos Sociales. España.

3. España. Real Decreto 298/2009, de 6 de marzo. Boletín Oficial del Estado, 7 de marzo de 2009, núm. 57, p. 23288.

4. Cerrolaja J, Mercé L, Pozuelo P, Jardón E. Orientaciones para la valoración del riesgo laboral y la incapacidad temporal durante el embarazo. Instituto Nacional de la Seguridad Social, Subdirección General de Coordinación de Unidades Médicas, España; 2008.

5. Guía de valoración de riesgos laborales en el embarazo y lactancia en trabajadoras del ámbito sanitario. ANMTAS. Ministerio de Ciencia e Innovación. Instituto da Salud Carlos III. España 2008. 